\title{
Blood cultures in sick children
}

\author{
H Lochan, ${ }^{1} \mathrm{MB}$ ChB, DCH (SA), FCPaed (SA); C Bamford, ${ }^{2} \mathrm{MB}$ ChB, DCH (SA), MPhil, FCPath (Micro), MMed (Med Micro); \\ B Eley, ${ }^{1} \mathrm{MB}$ ChB, FCPaed (SA), BSc (Hons) \\ ${ }^{1}$ Paediatric Infectious Diseases Unit, Red Cross War Memorial Children's Hospital, and Department of Paediatrics and Child Health,
University of Cape Town, South Africa
${ }^{2}$ National Health Laboratory Service, Groote Schuur Hospital and Division of Clinical Microbiology, University of Cape Town, South Africa
}

Corresponding author: H Lochan (harsha.lochan@uct.ac.za)

Background. Blood cultures (BCs) are frequently performed in sick children. A recent audit of BCs among adult patients documented high rates of contamination by coagulase-negative staphylococci (CoNS).

Objectives. To describe $\mathrm{BC}$ contamination rates and common pathogenic organisms causing bloodstream infection in children at a tertiarylevel children's hospital.

Methods. BC results for children admitted to Red Cross War Memorial Children's Hospital from 2008 to 2012 were extracted from the National Health Laboratory Service database. Pathogenic and non-pathogenic (contaminated) growth on BCs in children $<1$ year of age and $>1$ year of age, were analysed. Data analysis was performed using Epi Info version 3.5.1.

Results. A total of 47677 BCs were performed in the 5-year period. The proportion of contaminated specimens ranged between $5.9 \%$ and $7.2 \%$ per year $(p=0.4)$. CoNS was the predominant isolate in $53.8 \%$ of all contaminated BCs. Children $<1$ year of age experienced higher contamination rates than children $>1$ year of age $(8.7 \%$ v. $4.7 \%$; relative risk $1.84 ; 95 \%$ confidence interval (CI) $1.71-1.97)$. Pathogenic organisms were isolated in $6.2 \%$ (95\% CI 6.0 - 6.4) of all BC specimens. Among Gram-positive organisms, the proportion of Streptococcus pneumoniae isolates declined from $14.3 \%$ to $4.7 \%$ ( $p<0.00001)$, while there was a significant increase in Gram-negative organisms ( $51.8 \%-57.9 \%$; $p=0.04)$ over the 5 -year period. Klebsiella pneumoniae, the predominant Enterobacteriaceae isolated, decreased from $45.8 \%$ to $31.7 \%$ ( $p=0.004$ ).

Conclusion. This study identified unacceptably high BC contamination rates, emphasising the importance of collecting BC specimens under sterile conditions.

S Afr Med J 2013;103(12):918-920. DOI:10.7196/SAMJ.6979

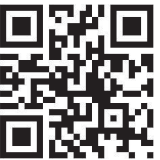

Blood cultures (BCs) are frequently performed in sick children either at initial presentation or during the course of hospitalisation following clinical deterioration. A positive $\mathrm{BC}$ result may help to determine the causative pathogen as well as guide appropriate antimicrobial therapy. ${ }^{[1]} \mathrm{A}$ recent audit of BCs among adult patients at G F Jooste Hospital, Cape Town documented high rates of contamination by coagulase-negative staphylococci (CoNS) over a 6-year period ranging from $4.6 \%$ to $9.3 \%$ per year. Furthermore, this study documented increasing antibiotic resistance of Gram-negative organisms. ${ }^{[2]}$

\section{Objective}

To understand $\mathrm{BC}$ contamination rates and the spectrum of pathogenic organisms causing bloodstream infection in children, we audited the results of BCs performed at our hospital. This analysis forms part of a series of studies on bloodstream infection at the hospital.

\section{Methods}

Study design

A retrospective audit of BCs. The Research Ethics Committee of the University of Cape Town approved the study. Because this was a retrospective analysis, informed consent was not obtained.

\section{Setting}

Red Cross War Memorial Children's Hospital (RCWMCH) is a 290bed tertiary hospital that serves the paediatric population of the City of Cape Town and the Western Cape Province and neighbouring provinces.

\section{Data extraction}

BCs performed at RCWMCH are processed at the National Health Laboratory Service (NHLS) microbiology laboratory at Groote Schuur Hospital (GSH). All BC results of specimens obtained at RCWMCH from 2008 until 2012 were extracted from the NHLS GSH database. All positive results were divided into recognisable pathogens and non-pathogens or contaminants, based on definitions established by the Centers for Disease Control and Prevention. ${ }^{[3]}$

\section{Statistical analysis}

The analysis included comparison of results, stratified according to age, i.e. $<1$ year and $\geq 1$ year of age. Data were analysed using Epi Info version 3.5.1. Conventional descriptive methods were used to analyse the data. The Mantel-Haenszel $\chi^{2}$ test was used to compare categorical data. Relative risk (RR) was used to estimate the size of differences. A $\chi^{2}$ test for linear trend was used to evaluate trends over time. A $p$-value of $<0.05$ was considered statistically significant.

\section{Results}

During the 5-year period there were 110938 admissions to the hospital, representing a mean \pm standard deviation of $22187.6 \pm 854.8$ admissions per year. A total of $47677 \mathrm{BC}$ specimens were taken, providing a $\mathrm{BC}$ specimen per hospital admissions ratio of 1:2.33.

The proportion of BCs with non-pathogenic growth (contaminated specimens) ranged between $5.9 \%$ and $7.2 \%$ per year. There was 
no significant trend in the frequency of contaminated specimens ( $\chi^{2}$ for trend $p=0.4$ ) (Table 1). The proportion of contaminated specimens was higher in children $<1$ year $(8.7 \%, 1928 / 22091$ v. $4.7 \%, 1215 / 25$ 586; RR 1.84; 95\% confidence interval (CI) 1.71 - 1.97). Non-pathogens were either Grampositive $(94.8 \%)$ or Gram-negative (5.1\%). CoNS was the predominant contaminant, accounting for $53.8 \%$ (1 792/3 143) of all nonpathogenic growth. Among contaminants, children $<1$ year experienced a higher rate of CoNS growth (63.5\% v. 46.7\%; RR 1.36; 95\% CI 1.27 - 1.46) (Table 1).

Overall, 6.2\% (2 969/47 677; 95\% CI for binomial proportion 6.0 - 6.4) of $\mathrm{BC}$ specimens yielded pathogenic organisms. The yield was lower in children $\geq 1$ year of age $\left(5.6 \%\right.$ v. $6.9 \%$; Mantel-Haenszel $\chi^{2}$ $p<0.00001$ ) (Table 1). The most common pathogens are shown in Table 2.

Gram-positive bacteria accounted for $36.5 \%$ (1 083/2 969) of all pathogenic isolates. The proportion of pathogens caused by Gram-positive bacteria, with Staphylococcus aureus and Streptococcus pneumoniae being those most commonly isolated, declined from $41.6 \%$ in 2008 to $33.4 \%$ in 2012 ( $\chi^{2}$ for trend $p=0.003)$. S. aureus and S. pneumoniae accounted for $13.7 \%$ (407/2 969) and $10.4 \%$ (309/2 969) of all pathogenic isolates, respectively. There was no significant change in the annual proportion of S. aureus isolates over the 5-year period ( $\chi^{2}$ for trend $p=0.2$ ). There was a progressive decline in the annual proportion of positive BCs due to S. pneumoniae, from $14.3 \%$ in 2008 to $4.7 \%$ in 2012 ( $\chi^{2}$ for trend $p<0.00001$ ). Enterococcus faecalis and E. faecium together were responsible for 5.2\% (153/2 969) of all pathogens with the annual proportion of these isolates increasing from $4.6 \%$ in 2008 to $7.3 \%$ in 2012 ( $\chi^{2}$ for trend $p=0.04$ ).

Gram-negative bacteria accounted for 56.6\% (1 679/2 969) of all pathogens (Table 2). The $\chi^{2}$ test for trend revealed a significant increase between 2008 and 2012 $(51.8 \%$ - $57.9 \%$; $p=0.04)$. Enterobacteriaceae dominated and accounted for $64 \%$ (1 074/1 679) of all Gram-negative pathogenic isolates and $36.2 \%$ (1 074/2 969) of all pathogens. There was no significant change in the annual proportion of Enterobacteriaceae ( $\chi^{2}$ for trend $p=0.76$ ). The

Table 1. Blood culture results according to age

\begin{tabular}{|c|c|c|c|c|c|}
\hline \multirow[b]{2}{*}{ Age (years) } & \multirow{2}{*}{$\begin{array}{l}\text { Total } \\
N\end{array}$} & \multicolumn{4}{|c|}{$\begin{array}{c}\text { Growth } \\
n(\%)\end{array}$} \\
\hline & & Positive & Pathogenic & Non-pathogenic & CoNS \\
\hline \multicolumn{6}{|l|}{ All } \\
\hline 2008 & 9396 & $1323(14)$ & $652(6.9)$ & $671(7.1)$ & $298(3.2)$ \\
\hline 2009 & 11187 & $1367(12.2)$ & 707 (6.3) & $660(5.9)$ & $311(2.8)$ \\
\hline 2010 & 10143 & $1338(13.2)$ & $652(6.4)$ & $686(6.8)$ & $358(3.5)$ \\
\hline 2011 & 9108 & $1217(13.4)$ & $557(6.1)$ & $660(7.2)$ & $429(4.7)$ \\
\hline 2012 & 7843 & $867(11.1)$ & $401(5.1)$ & $466(5.9)$ & $296(3.8)$ \\
\hline$p$-value ${ }^{*}$ & & 0.00007 & $<0.00001$ & 0.4 & $<0.00001$ \\
\hline \multicolumn{6}{|l|}{$<1$} \\
\hline 2008 & 4759 & $791(16.6)$ & $364(7.6)$ & $427(9)$ & $288(6.1)$ \\
\hline 2009 & 5241 & $776(14.8)$ & $368(7)$ & $408(7.8)$ & $205(3.9)$ \\
\hline 2010 & 4920 & $832(16.9)$ & $387(7.9)$ & $445(9)$ & $263(5.3)$ \\
\hline 2011 & 3968 & $652(16.4)$ & $261(6.6)$ & $391(9.9)$ & $279(7)$ \\
\hline 2012 & 3203 & $409(12.8)$ & $152(4.7)$ & $257(8)$ & $190(5.9)$ \\
\hline$p$-value & & 0.006 & 0.00001 & 0.7 & 0.008 \\
\hline \multicolumn{6}{|l|}{$>1$} \\
\hline 2008 & 4637 & $532(11.5)$ & $288(6.2)$ & $244(5.3)$ & $110(2.8)$ \\
\hline 2009 & 5946 & $591(9.9)$ & $339(5.7)$ & $252(5.2)$ & $106(1.8)$ \\
\hline 2010 & 5223 & $506(9.7)$ & $265(5.1)$ & $241(4.6)$ & $95(1.8)$ \\
\hline 2011 & 5140 & $565(10.9)$ & $296(5.8)$ & $269(5.2)$ & $150(2.9)$ \\
\hline 2012 & 4640 & 458 (9.9) & $249(5.4)$ & $209(4.5)$ & $106(2.3)$ \\
\hline$p$-value ${ }^{*}$ & & 0.2 & 0.1 & 0.8 & 0.1 \\
\hline
\end{tabular}

dominant Enterobacteriaceae isolates were Klebsiella pneumoniae (41.8\%), Escherichia coli (29.1\%) and Serratia marcescens (10.4\%). The annual proportion of Enterobacteriaceae infections caused by $K$. pneumoniae isolates decreased significantly from $45.8 \%$ in 2008 to $31.7 \%$ in 2012 ( $\chi^{2}$ for trend $p=0.004$ ). By contrast, an increasing trend was documented for $S$. marcescens from $3.4 \%$ in 2008 to $14.9 \%$ in 2012 ( $\chi^{2}$ for trend $p=0.0003$ ). The annual proportion of $E$. coli did not change significantly ( $\chi^{2}$ for trend $p=0.5$ ). Acinetobacter baumannii was responsible for 20.3\% (340/1 679) of all Gram-negative infections, isolates increasing annually from $12.4 \%(42 / 338)$ in 2008 to $25.2 \%(82 / 325)$ in 2011 ( $\chi^{2}$ for trend $p=0.0004$ ) and declining to $8.2 \%(19 / 232)$ in 2012.

Fungi constituted 6.7\% (200/2 969) of all pathogens, there being no significant change in the proportion annually $\left(\chi^{2}\right.$ for trend $p=0.1$ )

\section{Discussion}

This analysis provided initial information about $\mathrm{BC}$ results and bloodstream infection at RCWMCH. Of concern is the high contamination rates of $5.9-7.2 \%$ per year, which are above international target contamination rates of $2-3 \% \cdot{ }^{[4]}$ Similar high contamination rates were also documented in a recent adult study conducted in Cape Town. ${ }^{[2]}$ Because of technical challenges associated with drawing blood from infants, contamination was predictably higher in this subgroup. ${ }^{[5]}$ High contamination rates documented suggest that $\mathrm{BC}$ procedures were not standardised and sterility and optimal skin disinfection not uniformly practised. Standardisation of BC procedures is linked to improved pathogen yield when optimal blood volumes are collected. ${ }^{[1,6]} \mathrm{A}$ paediatric study completed in Kenya showed that the proportion of positive BCs increased with each additional millilitre of blood cultured. ${ }^{[7]}$ To limit contamination, all healthcare facilities should institute standardised guidelines for $\mathrm{BC}$ procedures and ensure ongoing training and education for existing and, especially, new medical staff.

In this study, only 1 in every $16 \mathrm{BC}$ specimens (6.2\%; 95\% CI 6.0 - 6.4) yielded a pathogenic isolate. Systematic reviews of bloodstream infections carried out in Africa and southeast Asia showed pathogen yields of $8.2 \%$ (3 527/43 130; 95\% CI 7.9 - 8.4) and $6.6 \%(1722 / 26258 ; 95 \%$ CI $6.3-6.9)$, respectively. ${ }^{[8,9]}$ The pathogen yield in this study was somewhat lower. One would expect a higher pathogen yield in our setting, as the aforementioned studies ${ }^{[8,9]}$ were a reflection of community-acquired bloodstream infections 
Table 2. Spectrum of common pathogens on blood culture

\begin{tabular}{llllll}
\hline & $\mathbf{2 0 0 8}$ & $\mathbf{2 0 0 9}$ & $\mathbf{2 0 1 0}$ & $\mathbf{2 0 1 1}$ & $\mathbf{2 0 1 2}$ \\
\hline Gram-positive, \% & & & & & \\
Total, $N$ & 271 & 255 & 234 & 190 & 133 \\
Streptococcus pneumoniae & 34.3 & 36.4 & 26.5 & 22.1 & 14.3 \\
Staphylococcus aureus & 33.9 & 32.2 & 42.3 & 40.5 & 42.1 \\
Streptococcus group B & 6.3 & 5.1 & 6.8 & 5.8 & 4.5 \\
Enterococcus faecium & 4.8 & 7.1 & 3.4 & 5.3 & 11.3 \\
Enterococcus faecalis & 6.3 & 7.5 & 4.7 & 14.7 & 10.5 \\
Gram-negative, \% & & & & & \\
Total, $N$ & 338 & 408 & 376 & 325 & 232 \\
Klebsiella pneumoniae & 31.3 & 27.2 & 27.9 & 21.2 & 22 \\
Escherichia coli & 21.9 & 19.1 & 17 & 14.5 & 22 \\
Acinetobacter baumannii & 12.4 & 17.2 & 17.8 & 25.2 & 8.2 \\
Serratia marcescens & 2.4 & 7.1 & 7.4 & 7.1 & 10.3 \\
Other, \% & & & & & \\
Total, $N$ & 43 & 43 & 43 & 42 & 36 \\
Candida albicans & 73.8 & 77.5 & 53.5 & 32.5 & 55.6 \\
Candida parapsilosis & 14.3 & 17.5 & 18.6 & 27.5 & 11.1 \\
Candida krusei & 4.7 & - & - & 11.9 & 19.4 \\
Candida lusitaniae & - & 5 & 4.7 & 2.4 & - \\
Candida tropicalis & 2.3 & - & 16.3 & - & 5.6 \\
Candida dubliniensis & - & - & 2.3 & 12.5 & - \\
Mycobacterium tuberculosis complex & 2.3 & 7 & - & 4.8 & 2.8 \\
& & & & & \\
& & & & &
\end{tabular}

only, whereas our study included both community-acquired and hospital-acquired bloodstream infections. Several factors, including prior antibiotic administration and suboptimal blood volumes, may have contributed to this lower yield. The Integrated Management of Childhood Illness Programme advises that any seriously ill child requiring urgent referral to hospital should receive intramuscular ceftriaxone prior to transfer. ${ }^{[10]}$ Many children referred from level 1 facilities would therefore have received parenteral antibiotics prior to $\mathrm{BC}$. Furthermore, the selection of patients for BCs should be reviewed to ensure that BCs are used optimally.

The dramatic decline in the culture of $S$. pneumoniae over time almost certainly reflects the effectiveness of the national pneumococcal immunisation programme and the declining paediatric HIV incidence. The 7-valent pneumococcal conjugate vaccine was introduced in April 2009 and in May 2011 was replaced with a 13-valent ving been discontinued (Profess

There are limitations to our study. Firstly, there was no analysis of the antimicrobial
Andrew Argent, personal communication). resistance patterns of the pathogens isolated, which will be the focus of a future study. Secondly, the focus of this study was BC results generated in a tertiary-level paediatric facility, rendering the findings of this audit, particularly with regard to pathogen trends, potentially inapplicable to other paediatric settings with different patient illness profiles and antimicrobial selection pressures. However, high contamination rates were also recorded in BCs in adult patients at G F Jooste Hospital, suggesting that the problem is widespread and that attention should be directed at optimising $\mathrm{BC}$ procedures according to recently published recommendations. ${ }^{[1]}$ Thirdly, due to the retrospective nature of the study, $\mathrm{BC}$ specimens with inadequate volumes could not be evaluated.

In conclusion, this study identified unacceptably high contamination rates, emphasising the importance of collecting BC specimens under sterile conditions. Decreasing trends in Gram-positive organisms, in particular S. pneumoniae, and an increase among the Gram-negative group of organisms were demonstrated.

\section{References \\ 1. Ntusi N, Aubin L, Oliver S, Whitelaw A, Mendelson M. Guideline for the optimal use of blood cultures. S Afr Med 2010;100(12):839-843 \\ 2. Kenyon CR, Fatti G, Schrueder N, Bonorchis K, Meintijes G. The value of blood culture audits at peripheral hospitals. S Afr Med J 2012;102(4):232-233. \\ 3. Centers for Disease Control and Prevention, and the National Healthcare Safety Network. CDC/NHSH Surveillance Definition of Healthcare-Associated Infection and Criteria for Specific Types of Infection in the Acute Care Setting. Atlanta: CDC, 2013 http://www.cdc.gov/nhsn/pdfs/pscmanual/17pscnosinfdef current.pdf (accessed 26 February 2013). \\ 4. Hall KK, Lyman JA. Updated review of blood culture contamination. Clin Microbiol Rev 2006;19(4):788-802. [http:// dx.doi.org/10.1128/CMR.00062-05 \\ 5. Pavlovsky M, Press J, Peled N, Yagupsky P. Blood culture contamination in pediatric patients: Young children and young doctors. Pediatr Infect Dis 2006;25(7):611-614. [http://dx.doi org/10.1097/01.inf.0000220228.01382.88] \\ 6. Connell TG, Rele M, Cowley D, Buttery IP Curtis N. How reliable is a negative blood culture result? Volume of blood sabmited is a negative blood culture $\mathrm{D}$, $\mathrm{Bull}$ ? Volume or blood submitte for culture in routhe practice in a children's hospital. Pediatrics . . Berkley JA, Lowe BS, Mwangi I, et al. Bacteraemia among children admitted to a rural hospital in Kenya. N Engl J Me 2005;352:39-47. [http://dx.doi.org/10.1056/NEJMoa040275] \\ 8. Reddy A, Shaw AV, Crump JA. Community-acquired bloodstream infections in Africa: A systematic review and meta- analysis. Lancet Infect Dis 2010;10(6):417-432. [http://dx.doi. org/10.1016/S1473-3099(10)70072-4] \\ 9. Deen J, von Seidlein L, Andersen F, Elle N, White NJ, Lubell Y. Community-acquired bacterial bloodstream infections in developing countries in south and southeast Asia: A systematic review. Lancet Infect Dis 2012;12(6):480-487. [http://dx doi. org/10.1016/S1473-3099(12)70028-2 \\ 10. South African Department of Health. Integrated Management of Childhood Ilness Guidelines. Pretoris Depagent of Health, 2010.}

